Relations industrielles

Industrial Relations

\title{
L'origine de la formule Rand
}

\section{Gérard Dion}

Volume 30, numéro 4, 1975

URI : https://id.erudit.org/iderudit/028662ar

DOI : https://doi.org/10.7202/028662ar

Aller au sommaire du numéro

\section{Éditeur(s)}

Département des relations industrielles de l'Université Laval

ISSN

0034-379X (imprimé)

1703-8138 (numérique)

Découvrir la revue

\section{Citer cet article}

Dion, G. (1975). L'origine de la formule Rand. Relations industrielles / Industrial Relations, 30(4), 747-760. https://doi.org/10.7202/028662ar
Résumé de l'article

Trente ans se sont écoulés depuis qu'à l'occasion de la grève chez Ford Motor à Windsor, le juge Ivan C. Rand a rendu une décision arbitrale célèbre. L'auteur retrace l'origine de la formule Rand et montre comment celle que Von connaît aujourd'hui a été émasculée de certains de ses éléments essentiels.
Tous droits réservés @ Département des relations industrielles de l'Université Laval, 1975
Ce document est protégé par la loi sur le droit d'auteur. L’utilisation des services d'Érudit (y compris la reproduction) est assujettie à sa politique d'utilisation que vous pouvez consulter en ligne.

https://apropos.erudit.org/fr/usagers/politique-dutilisation/ 


\section{L'origine de la formule Rand}

\section{Gérard Dion}

Trente ans se sont écoulés depuis qu'à l'occasion de la grève chez Ford Motor à Windsor, le juge Ivan C. Rand a rendu une décision arbitrale célèbre. L'auteur retrace l'origine de la formule Rand et montre comment celle que l'on connait aujourd'hui a été émasculée de certains de ses éléments essentiels.

Parmi les conflits du travail qui ont eu un impact sur les relations du travail dans l'ensemble du Canada, la grève des travailleurs de Ford Motor Company à Windsor, Ontario, en 1945 occupe une place tout à fait particulière en raison des causes qui lui ont donné naissance, du nombre de travailleurs concernés, des événements qui se sont déroulés en cours de grève et surtout en raison de la façon dont elle a été réglée.

Le 12 septembre $1945,9,500$ ouvriers, membres de la section locale 200 des Travailleurs unis de l'automobile (TUA), à la suite de négociations infructueuses, décident de déclencher une grève qui durera jusqu'au 20 décembre suivant alors que les parties acceptent le projet conjoint de règlement proposé par les gouvernements fédéral et provincial prévoyant l'arbitrage par un juge de la Cour suprême du Canada des points qui ne pourraient être réglés par négociations collectives. C'est le juge Ivan $C$. Rand qui fut chargé de cette mission et la question qui opposait radicalement les parties touchait la sécurité syndicale. Des séances d'audition furent tenues du 9 au 15 janvier 1946 et la décision a été rendue le 29 janvier.

La guerre, qui venait de se terminer un mois auparavant, avait amené toutes sortes de restrictions à l'action libre des syndicats. C'était le premier conflit d'envergure qui surgissait depuis la fin des hostilités. La Loi des mesures de guerre n'avant pas encore été rappelée, l'industrie de l'automobile continuait à relever de la juridiction fédérale.

DION, G., professeur, Département des relations industrielles, Université Laval, Québec. Boursier Killam 1976. 
Les relations du travail chez Ford à Windsor n'avaient jamais été très harmonieuses. Durant la guerre, on y avait connu une multitude de grèves sauvages. C'était avec beaucoup de réticences que la compagnie avait accepté de négocier avec le syndicat représentant ses employés. La cause majeure de mécontentement était que pour les travailleurs de Windsor, malgré la proximité des États-Unis et le fait que des deux côés de la frontière on avait le même syndicat et la même compagnie, la convention collective au Canada était loin d'accorder les mêmes conditions et les mêmes avantages dont jouissaient leurs confrères des États-Unis. Mais ce qui était au coeur de l'affrontement, c'était surtout la demande de la part du syndicat de l'atelier syndical avec la retenue obligatoire des cotisations et l'établissement d'une procédure de règlement des griefs.

Traumatisée par les grèves sauvages qu'elle avait subie et aussi par l'orientation radicale dont elle accusait les dirigeants syndicaux de l'époque, la compagnie s'opposait farouchement à accorder toute forme de sécurité syndicale au nom de la liberté de travail et de la liberté d'association. De leur côté, les syndicats soupçonnaient dans cette attitude un moyen camouflé d'affaiblir leur puissance de négociation et ainsi de perpétuer en situation d'infériorité les travailleurs canadiens. De part et d'autre, on en faisait une affaire de principe. D'ailleurs, à cette époque, aux États-Unis comme au Canada, dans les rapports entre syndicats et employeurs, la question de sécurité syndicale était devenue un symbole de l'acceptation ou du refus du syndicalisme.

\section{L'IMPORTANCE DE LA SÉCURITÉ SYNDICALE}

Le régime légal des relations du travail établi par l'arrêté en conseil C.P. 1003 en 1944 avait indirectement contribué à donner une importance particulière à la sécurité syndicale. Les syndicats avaient toujours cherché à s'assurer l'adhésion des travailleurs et à les amener à demeurer membres de l'organisation tant par leurs propres moyens grâce aux différentes caisses de secours ou d'assurance que par l'introduction dans les conventions collectives des clauses de sécurité syndicale.

Selon le nouveau régime juridique destiné à protéger l'exercice de la liberté d'association, il n'était plus loisible à l'employeur de refuser de reconnaître un syndicat comme représentant ses employés, ce pouvoir relevant désormais d'un organisme public qui accorde l'accrédita- 
tion lorsque le syndicat groupe la majorité des travailleurs de l'unité de négociation. En contrepartie, l'exercice du droit de grève était réglementé. Toute grève devenait illégale aussi longtemps que n'avaient pas été épuisées toutes les étapes de la procédure établie par la loi dont la première était l'obtention de l'accréditation. C'est donc dire qu'un employeur, pour négocier ou renouveller une convention collective pouvait toujours exiger du syndicat une représentation majoritaire. Le syndicat, de son côté, s'il voulait avoir les coudées franches et pouvoir recourir à la loi, devait maintenir un effectif dépassant la majorité des travailleurs de l'unité de négociation. Ainsi, l'arrêté C.P. 1003 destiné à protéger les syndicats les contraignait à prendre les moyens de conserver leurs membres et même de les accroître. La sécurité syndicale est alors devenue un enjeu généralisé dans toutes les négociations collectives.

Les résultats d'une compilation effectuée par le ministère fédéral du travail sur les litiges portés devant une commission de conciliation indiquaient clairement l'importance de la sécurité syndicale.

\begin{abstract}
«Depuis l'établissement des Règlements des relations ouvrières en temps de guerre, en mars 1944, jusqu'au 31 décembre 1945, 147 commissions de conciliation ont été instituées dont 132 ont fait rapport à date. Des 132 commissions de conciliation qui ont fait rapport, 125 ont eu à s'occuper de la question de la sécurité syndicale, et $90(72 \%)$ ont recommandé un mode quelconque de sécurité syndicale. Par conséquent, cinq sur sept commissions de conciliation qui ont eu à s'occuper de la question ont recommandé la sécurité syndicale $\gg 1$.
\end{abstract}

À la même époque, nous avions fait un relevé de toutes les conventions collectives négociées et déposées dans la province de Québec entre le 2 février 1944 et le 20 avril 1946 sous l'empire de la Loi des relations ouvrières, l'équivalent québécois au C.P. 1003. Nous y avions constaté que $58.6 \%$ des conventions comportaient une clause quelconque de sécurité syndicale. La disposition la plus en vogue était l'atelier syndical parfait ${ }^{2}(27 \%)$ et ensuite venait la retenue des cotisations syndicales ${ }^{3}$.

1 Gazette du travail (Ottawa), vol. 46, no 2, fév. 1946, p. 149.

2 Tous les employés compris dans l'unité de négociation doivent, pour demeurer en emploi, être membres du syndicat.

3 Gérard DION, "Clauses de sécurité syndicale dans les conventions collectives de la province de Québec », Bulletin des relations industrielles, (Québec), vol. 2 , no 2 , p. 5 . 


\section{LA GRÈVE}

La grève déclenchée chez Ford Motor en est venue avec le temps à prendre l'allure d'un affrontement entre le patronat canadien et le syndicalisme sur cette question de la sécurité syndicale que des deux côtés on jugeait comme primordiale.

$\mathrm{Si}$, au niveau de la direction du syndicat international et du Congrès canadien du travail on exprimait certaines réticences vis-à-vis de la direction locale manipulée par des éléments communistes et des méthodes utilisées ${ }^{4}$, on appuyait les objectifs poursuivis par la section 200 des TUA de l'usine Ford qui manifestait une solidarité enthousiaste et jouissait de la sympathie de l'ensemble de la population de la ville de Windsor.

Pendant le premier mois la grève s'est déroulée sans incident grave en dehors du piquetage illégal des bureaux de la compagnie. Au début de novembre, certains événements sont venus lui donner une envergure provinciale et canadienne. Devant l'impasse des négociations et l'absence de solution en vue, la direction locale qui jusque là avait respecté une entente tacite, selon laquelle on avait laissé les agents de sécurité et les travailleurs affectés à la maintenance de la centrale d'énergie assumer leurs services, décida de dresser une ligne de piquetage autour de la centrale. La Commission de police, à la demande de l'Association des assureurs et contre le gré des autorités municipales, détacha un certain nombre de policiers pour briser cette ligne de piquetage.

L'échec de cette initiative amena la Commission de police et l'Association des assureurs à faire appel à $\mathbf{M}$. Blackwell, procureur général de l'Ontario, pour obtenir l'aide de la police provinciale. On craignait, disaient-ils, l'éventualité de dégats de l'ordre de $\$ 23$ millions et on ne voulait pas en être tenu responsable. Le premier ministre de l'Ontario, George Drew, à son tour, demanda au premier ministre du Canada, W. L. MacKenzie King, la collaboration de la gendarmerie royale. Paul Martin, secrétaire d'État, qui était député de Windsor, s'est vainement opposé au Cabinet considérant pareille intervention comme une insulte aux grévistes et à leurs sympathisants.

4 Dans son ouvrage, Nationalism, Communism, and Canadian Labor, Toronto, University of Toronto Press, 1973, pp. 142-145, Irving Martin ABELLA expose bien le rôle joué par les éléments communistes dans cette grève. 
Le 3 novembre, le détachement de la police provinciale d'Ontario et celui de la gendarmerie royale en arrivant à Windsor se trouvèrent en face d'un embouteillage monstre causé par 1500 automobiles bloquant sur un parcours de cinq rues l'accès à l'usine Ford no 1 . Ce n'est pas sans difficulté que les dirigeants syndicaux réussirent à la fin à amener les manifestants à obtempérer à un ordre formel venant du procureur général de libérer la circulation.

Deux jours plus tard, défiant les directives de leurs dirigeants internationaux, les 8,500 membres de la section 195 des Travailleurs unis de l'automobile comprenant les usines Chrysler, à la suite d'un vote secret unanime, déclenchaient une grève de sympathie qu'ils maintiendront jusqu'au 30 novembre. Le comité de grève adressa plusieurs centaines de télégrammes à divers syndicats leur demandant de faire une grève générale d'une journée à travers le pays. Condamné par le Congrès canadien du travail, ce geste n'a pas donné de résultat.

L'impasse était complète et l'affaire avait pris une proportion telle qu'elle donna lieu à des débats à la Chambre des Communes. Les députés CCF demandaient une intervention directe du Parlement. Ils suggérèrent d'abord une législation spéciale rendant l'atelier syndical obligatoire. Comme on prévoyait que la Loi des mesures de guerre devait bientôt prendre fin et que le gouvernement provincial reprendrait sa juridiction complète en matière du travail, le problème ne serait réglé que temporairement. S'inspirant de la législation américaine sur les chemins de fer, ils revinrent à la charge en préconisant la mise en tutelle de la compagnie Ford au Canada. Le gouvernement a étudié ce projet, mais comme il était fort probable que la compagnie connaitrait des pertes d'exploitation pour l'année en cours, on se demandait qui assumerait ces pertes? On a enfin considéré la possibilité de recourir à une loi spéciale imposant le retour au travail et l'arbitrage obligatoire du conflit. Le ministre du travail, Humphrey Mitchell était très hésitant devant une mesure semblable et préféra l'utilisation d'un moyen plus traditionnel: la conciliation. Il pensait qu'au lieu d'imposer une solution qui ne serait agréable à l'une ou l'autre des parties et qui mettrait le feu aux poudres dans les relations du travail partout au Canada, il valait mieux les amener à un compromis satisfaisant qui pourrait à son tour créer un précédent et éviter des conflits dans les autres industries.

Le travail de conciliation fut long et pénible. À trois reprises, on fut sur le point de se mettre d'accord, mais au dernier moment, les 
représentants syndicaux dans des déclarations fracassantes rejettaient publiquement le compromis qu'ils avaient préalablement laissé entendre comme leur étant acceptable. Il importait alors d'amener à la table de négociation des interlocuteurs sérieux et responsables. Paul Martin se rendit aux États-Unis discuter l'affaire avec les instances supérieures des syndicats internationaux et George Addes, vice-président international des TUA fut mandé au Canada pour étudier la situation. Il constata que des activistes communistes sabottaient toute tentative de règlement. Avec Pat Conroy, secrétaire du Congrès canadien du travail, il prit l'affaire en main. Ils réussirent à convaincre les travailleurs de retirer la ligne de piquetage devant les bureaux et la centrale d'énergie et les négociations recommencèrent dans une autre atmosphère. Les travailleurs par ailleurs avaient perdu confiance dans leurs dirigeants locaux et étaient fatigués de la grève. C'est alors que les deux parties acceptèrent la suggestion de s'en remettre à l'arbitrage d'un juge de la Cour suprême du Canada et la grève prit fin le 20 décembre.

\section{L'ARBITRAGE DU JUGE RAND}

Le juge Ivan C. Rand jouissait de la réputation d'être un homme sage, pondéré, ouvert et progressiste. Il mena l'affaire avec une célérité peu coutumière. Des séances d'auditions furent tenues du 9 au 15 janvier 1946 et la décision a été rendue le 29 janvier.

L'importance de cette décision provient surtout de l'originalité des conclusions, mais encore des motifs sur lesquels elle est fondée.

Le juge Rand y développe toute une philosophie des relations du travail dans une société industrielle et démocratique. Cette synthèse par sa clarté et sa précision est un véritable classique qui, conserve encore aujourd'hui sa valeur et son actualité. Comme il est difficile de se procurer ce texte, nous le reproduisons intégralement en annexe 5 .

Après des considérations générales sur les exigences d'une société démocratique qui doit tenir compte des droits individuels et des devoirs sociaux; de la nécessité du pouvoir tenu en alerte par une opinion

5 Le texte de la décision a été publié intégralement dans la Gazette du travail, (Ottawa), vol. 46, no 1, janv. 1946, pp. 126-134, mais la traduction française est si mauvaise qu'elle a dû être refaite à partir du texte anglais paru dans The Labour Gazette, vol. 46, no 1, janvier 1946. 
publique éclairée et contrôlée par l'exercice du droit de vote, le juge Rand envisage la nature de l'entreprise avec les droits de la direction, la nature des syndicats avec leur rôle; le genre de relations qui doivent s'établir entre ces deux institutions et les principes sur lesquels on doit se guider.

Les entreprises sont nécessaires au développement et au progrès de l'économie, mais on doit s'élever contre une conception absolutiste du droit de propriété qui donnerait un pouvoir sur les personnes.

Le droit d'association est un droit naturel. Les syndicats sont nécessaires pour représenter les travailleurs et contrebalancer le pouvoir patronal. Afin d'exercer leur rôle d'une façon efficace, ils doivent être forts et stables. Ils doivent encore être responsables.

Au-dessus des divergences d'intérêt entre syndicat et entreprise, il existe entre les deux une communauté d'intérêts qui leur permet d'accomplir leur mission au sein de l'économie. L'un et l'autre sont redevables à la société de leur comportement dans les conflits qui les opposent.

Syndicalisme et négociation collective sont devenus des institutions sociales acceptées comme désirables par l'ensemble de la société et ainsi reconnus par la législation. On ne doit pas retourner en arrière.

Les forces susceptibles de contribuer à affaiblir le syndicat ou à le détruire peuvent venir de l'employeur ou des travailleurs eux-mêmes. La loi pourvoit déjà à des mesures destinées à protéger le syndicat contre l'action de l'employeur, mais il n'y a rien pour empêcher l'égoïsme des travailleurs et c'est ce qui rend la sécurité syndicale indispensable. Cette sécurité syndicale, cependant, ne doit pas mettre l'employeur à la merci du syndicat dans l'embauchage ni permettre au syndicat d'exercer un despotisme sur les travailleurs. Il faut donc essayer de concilier la liberté individuelle des travailleurs et leur devoir de solidarité.

Puisque tous les travailleurs dans un établissement bénéficient des avantages que leur procure le syndicat, il est normal que tous contribuent à défrayer les coûts de son fonctionnement pour ses activités proprement syndicales. S'ils ne veulent pas devenir membres du syndicat, on doit respecter leur désir, mais ils devront quand même lui verser l'équivalent de la cotisation syndicale ordinaire. L'employeur fera sa part en retenant sur les salaires de tous les travailleurs assujettis à la convention ces montants qu'il remettra ensuite au syndicat. 
Il est évident que les travailleurs qui s'abstiendront d'adhérer au syndicat n'auront pas le droit de participer à la gestion des affaires internes du syndicat. Mais, advenant le cas où le syndicat voudrait déclarer une grève, un vote devrait être obligatoirement tenu par bulletin secret sous la surveillance d'un fonctionnaire du ministère du travail de l'Ontario et une majorité devrait être obtenue. Comme ce sont tous les travailleurs qui sont affectés par cette cessation de travail, membres ou non du syndicat, les non membres auront aussi le droit de participer à ce vote.

Enfin, comme la puissance du syndicat était accrue, le juge Rand considérait qu'il devait devenir responsable des gestes qu'il poserait ainsi que de ceux de ses membres. Et pour l'y aider, il prévoyait une sanction dans le cas de grève déclenchée par le syndicat sans avoir obtenu un vote majoritaire préalable ou de grève sauvage déclenchée par un groupe de syndiqués que le syndicat n'aura pas répudiée. Cette sanction consistait dans la cessation de tout précompte syndical par l'employeur pendant un minimum de deux mois jusqu'à un maximum de six mois dans le premier cas et, dans le second cas, durant un minimum d'un mois jusqu'à un maximum de quatre mois. Au-delà du minimum fixé, l'employeur pouvait se rendre au maximum, mais si le syndicat trouvait la sanction trop sévère, l'affaire devait être soumise à un arbitre dont la décision sera finale.

Des sanctions étaient aussi prévues contre chacun des travailleurs eux-mêmes participant à une grève inautorisée ou à une suspension de travail concertée autrement que sur l'ordre du syndicat. Elles consistaient dans une amende de $\$ 3.00$ par jour pour chaque jour d'absence et dans la perte d'un an d'ancienneté pour absence ininterrompue pendant une semaine complète ou partielle.

\section{LES RÉACTIONS DE LA DÉCISION RAND}

À la suite de la publication de la décision Rand sur la sécurité syndicale, que certains journaux ont qualifiée de révolutionnaire, les réactions chez les parties intéressées furent limitées et réservées. Personne n'entonna le chant de la victoire.

Le président de la société Ford s'est contenté de dire: «It is our feeling that the agreement provides the basis for a good relationship

6 Cité par Stewart CRYSDALE, The Industrial Struggle and Protestant Ethics in Canada, Toronto, The Ryerson Press, 1961, p. 62. 
between the Company and its employees ». ${ }^{6}$ Quant au président du Congrès canadien du travail, Aaron R. Mosher, auquel les TUA étaient affiliés, selon un rapport de la Presse canadienne, il a exprimé un accord réservé en ajoutant cependant que la décision apportait une amélioration et qu'elle était un bon équivalent de l'atelier syndical ${ }^{7}$.

Chez les autres centrales syndicales, le président du Congrès des métiers et du travail du Canada, Percy Bengough, tout comme la revue de ce groupement, le Trade and Labor Congress Journal, ont ignoré complètement la décision. Le président de la Confédération des travailleurs catholiques du Canada, Alfred Charpentier, dans une déclaration publiée dans le journal officiel de son groupement disait:

«Pour la première fois, cette décision reconnaît à l'organisation ouvrière le droit de toucher une cotisation de reconnaissance pour services rendus. Par ailleurs, elle laisse à l'ouvrier sa liberté d'appartenir ou non à l'union signataire du contrat de travail dans l'usine où lui-même est occupé. Nous considérons cette décision bonne dans les deux cas. Cependant, elle n'est pas parfaite : elle ne dit pas si cet ouvrier a la liberté d'appartenir à une autre organisation de son choix. La décision Rand influera considérablement sur la législation syndicale du pays et aidera de beaucoup à la perfectionner ${ }^{8}$.

Dans un article d'une extrême violence dont le titre lui-même «une supercherie malfaisante » était déjà très significatif, l'éditorial de la Gazette de Montréal condamnait péremptoirement la décision en énonçant des objections qui seront par la suite les arguments resassés des milieux patronaux hostiles.

"The compromise which Mr Justice Rand purports to lay down in his decision on the Ford strike issue is illusory in principle and misleading as to its specific effect.

On close analysis the freediom actually assured is hollow and fictitious, and is associated with some entirely new and objectionable principles in the sphere of labor relations.

The proposal that workers shall be compelled to pay union dues las a condition of employment but at the same time remain outside union membership is a negation of the principle which has been the bedrock of British liberties for centuries - no taxation without representation. The fact that employees are free to join the union if

7 The Gazette, Montréal, 30 janvier 1946.

8 Le Travail, Montréal, mars 1946. 
they wish does not alter the basic fact that the Rand ruling requires the company to levy union dues from the pay of those who may not wish to join $\gg$.

Saturday Night, sous la signature de D.P. O'Haern, exprime un autre son de cloche. Pour lui, la décision Rand est vraiment un pas en avant dans les relations du travail. Il s'explique les réticences des syndicats et des employeurs, mais il prévoit que lorsqu'on l'aura comprise, il est probable qu'elle se généralisera et servira de modèle.

«First labor reaction to the Rand report has had its elements of opposition. This is not cause for great concern. Labor leadership is naturally "opposed". It has had to be by nature in its long fight for labor aims. And it is always very slow to make up its mind to new turns of events (recall the opposition in the early days of the baby bonus). When it has had time to digest the Rand proposals it should be well satisfied.

One early objection to the Rand formula on the part of labor is the strike vote provision giving all workers a vote and requiring a simple majority of all coming under the contract. Aside from the standpoint that there is only a very shaky leg to stand on here, the objection is one that with time should dissolve. There is no reason why under the Rand conditions a good union shouldn't attract all but a small minority of the workers. And if it doesn't have them the implication is that perhaps it would be better if its strike vote were defeated $\gg 10$.

La revue officielle du Congrès canadien du travail dans son numéro de février 1946, tout en exprimant certaines réserves, se montre très optimiste. "Probably no recent event in Canada has aroused so much attention; the enunciation of the formula may prove to be a turning point in the history of Labour relations in Canada ». On passe sous silence l'obligation de prendre un vote de grève sous contrôle extérieur ainsi que le droit des non membres à y participer pour s'arrêter à des questions qui, somme toute, sont secondaires.

«There are one or two dangers inherent in the Rand formula. It is conceivable that an anti-union management might deliberately stir up so much irritation among a group of workers that an unauthorized strike would occur. However, the provision for an umpire is likely to provide sufficient protection to the union in this

9 «Ford Decision a Mischievous deceit», The Gazette (Montréal), 30 janvier 1946.

10 «Rand Award Really Moves Labor Relations Ahead», Saturday Night, (Toronto), 9 février 1946. 
respect. The distinction between dues-payers and members may also cause some confusion and misunderstanding. It may be supposed that workers who are paying dues in any event may feel that they might just as well join the union and participate in its activities. On the other hand, there might be less rather than more interest in the work of the union under the new plan, and the union will have to meet this by expending a larger portion of its assured income in publishing educational and other material which will be available to all employees $\gg 11$.

Sauf rarissismes exceptions, la plupart des groupements patronaux adoptèrent une attitude extrêmement réticente et même plutôt hostile à la décision Rand. La position officielle de l'Association professionnelle des industriels du Québec peut à cet égard être considérée comme typique.

«Par la taxe prélevée sur le salaire de tous les employés, le juge Rand prétend concilier la liberté syndicale à la sécurité syndicale. Il oublie le principe anglais de finances publiques: "No taxation without representation ». Verser une somme à un Syndicat sans voix au chapitre est une façon polie mais réelle de vous obliger à rentrer dans ce Syndicat. Et cette taxe imposée devient alors un camouflage d'atelier fermé.

Sans doute l'ouvrier non syndiqué profite des activités syndicales, mais à la suggestion de M. J. C. Cameron, professeur à l'Université Queen, ne faudrait-il pas, au moyen des contributions soutirées des non syndiqués, constituer un fond indépendant sous la surveillance d'une commission indépendante qui verrait à employer les argents ainsi accumulés à défrayer les dépenses du contrat collectif? Cette proposition nous paraît beaucoup plus dans l'ordre, car confier à un Syndicat non incorporé l'administration d'une somme importante sans le contrôle des intéressés nous semble une violation de la liberté démocratique.

Cette décision laisse entendre que l'augmentation de salaire est l'apanage exclusif du Syndicat sans lequel un patron, conscient de ses responsabilités sociales, ne pourrait élever, de son chef, le barème de ses salaires.

De plus, cette décision va à l'encontre de nos lois civiles, et il est antisocial de taxer un salarié sans son autorisation expresse en faveur d'un organisme qui n'est pas un corps public.

Ce malencontreux impôt vicie par ailleurs la notion même de la liberté syndicale. Pour maintenir une telle innovation de principe louvrier devra payer une double cotisation: la cotisation du Syndicat imposé et la cotisation du Syndicat qu'il choisira librement. 
D'autre part, avons-nous songé aux luttes ouvrières que cette décision pourra susciter sur le plan syndical? En effet, quel est le Syndicat qui ne sera pas tenté par l'appât d'un revenu annuel de cent quatorze mille dollars comme dans le cas que nous fournit les 9,500 ouvriers de la Ford Motor Company de Windsor, Ontario. Voilà, parmi tant d'autres raisons, des motifs d'ordre social, économique et légal qui obligent l'Association professionnelle des industriels à désapprouver la retenue syndicale obligatoire telle que proposée par le juge Rand $\gg 12$.

\section{L'ÉVOLUTION DE LA FORMULE RAND}

La décision Rand a suivi un cheminement assez inattendu. Dans l'exposé de ses motifs, le juge Rand avait soutenu qu'il importait de s'attaquer aux problèmes de fond qui amenaient les conflits. Par ailleurs, en proposant sa formule de règlement dans le cas qui lui avait été confié, il prenait la peine d'avertir qu'il ne la croyait pas nécessairement applicable partout en toutes circonstances. «Je ne prétends pas un instant, devrais-je peut-être ajouter, que l'on puisse appliquer ce principe (retenue des cotisations syndicales chez tous les travailleurs de l'unité de négociation) d'une manière générale ».

La décision comprenait trois volets que son auteur voulait expressément indissociables :

a) la retenue par l'employeur des cotisations syndicales ordinaires sur la paye de tous les travailleurs assujettis à la convention, qu'ils soient membres ou non du syndicat;

b) l'obligation pour le syndicat de tenir, sous contrôle extérieur, avant le déclenchement de toute grève un vote par bulletin secret auquel ont droit de participer tous les travailleurs de l'unité de négociation, qu'ils soient membres ou non du syndicat;

c) des sanctions spécifiques imposées au syndicat et aux travailleurs se livrant à une grève illégale ou qui n'aurait pas rempli les conditions précédentes.

Ce que l'on désigne aujourd'hui par formule Rand ne correspond qu'au premier volet et va absolument à l'encontre du texte de la décision et de l'esprit dans lequel son auteur l'a rendue.

Comment expliquer qu'avec le temps on ait laissé tomber deux éléments essentiels: la tenue obligatoire du vote de grève auquel le

12 Le Devoir, (Montréal), 6 avril 1946. 
non syndiqué a le droit de participer et les sanctions dans le cas de grèves illégales? C'était ces deux éléments qui contribuaient à contrebalancer le pouvoir économique accordé au syndicat et à le rendre démocratique et responsable.

Plusieurs hypothèses peuvent être avancées. Nous croyons que les grands responsables de cette émasculation de la formule Rand originale sont les employeurs eux-mêmes. Leur manque de vision les a empêchés d'envisager et de comprendre tous les effets positifs que comportait la formule Rand originale. En s'y opposant globalement d'une façon inconsidérée et en ne tenant pas compte de la part de droits accordés aux non syndiqués ni du fardeau de responsabilité imposé aux syndicats, ils ont laissé à ceux-ci l'initiative d'user de leur pouvoir de négociation pour obtenir le genre de sécurité syndicale qu'ils désiraient. Par ailleurs, il ne faut pas être surpris que les syndicats n'aient pas insisté pour que dans les conventions collectives soient insérées des clauses limitant leur liberté absolue d'action et les rendant susceptibles de sanctions.

C'est ainsi que les syndicats ont fait des choix parmi les principes énoncés par le juge Rand et n'ont conservé de la décision que la retenue obligatoire des cotisations syndicales chez tous les travailleurs de l'unité de négociation membres ou non du syndicat.

Dans l'esprit du juge Rand, sa décision devait inciter, les non syndiqués à joindre les rangs du syndicat puisqu'étant déjà obligés à payer les cotisations il ne leur en coûterait pas davantage pour être membres à part entière. Il n'y a pas eu d'enquête sur ce sujet et nous ne savons pas dans quelle mesure ce résultat a été atteint.

Dans son petit ouvrage sur l'histoire du syndicalisme au Canada, l'ancien directeur de l'OFL Labour Review rapporte:

«It has been said that the Ford strike for union security did more to put industrial unionism on its feet than any other single event.

As for UAW Local 200, by 1955 it had a full union shop at Fordi $\gg 13$.

La convention collective en vigueur depuis décembre 1973 entre la société Ford et l'union internationale des travailleurs unis de l'automobile contient à la fois une disposition d'atelier syndical imparfait ${ }^{14}$

13 Morden LAZARUS, Years of Hard Labour, Toronto, Ontario Federation of Labour, 1974, p. 57.

14 Tous les travailleurs déjà membres du syndicat doivent le demeurer pour conserver leur emploi et les nouveaux embauchés doivent joindre les rangs du syndicat. 
et la retenue obligatoire des cotisations chez tous les travailleurs syndiqués ou non. Le syndicat s'engage à ne pas faire de grève sans avoir épuisé la procédure de règlement des griefs et sans en avoir obtenu l'autorisation $\mathrm{du}$ conseil exécutif du syndicat international et qu'une copie de cette autorisation ait été transmise à la compagnie. Advenant le cas où il y aurait violation de ces dispositions, la compagnie aura le droit de mettre fin à la convention collective ou d'imposer aux travailleurs des sanctions disciplinaires qui pourront faire l'objet d'un grief à être traité selon la procédure générale établie dans la convention ${ }^{15}$. Il n'est donc pas question spécifiquement de la cessation de la retenue syndicale ni d'amendes précises ou de perte d'ancienneté pour les travailleurs, comme l'imposait la décision Rand de 1946.

Trente ans se sont écoulés depuis cette décision célèbre. On vient de voir quelles transformations a connues le modèle original. Il existe pas de statistiques complètes permettant de connaître toute l'extension de la formule Rand aujourd'hui à travers le Canada. On possède cependant certaines données qui sont assez révélatrices.

En 1974, selon un relevé effectué par Travail Canada dans les établissements de l'industrie manufacturière possédant 500 travailleurs et plus, $29.9 \%$ des conventions collectives contenaient une clause de formule Rand et ceci visait $32.4 \%$ des travailleurs. Par ailleurs, $67.9 \%$ des conventions collectives couvrant $64.4 \%$ des travailleurs possédaient une autre forme de retenue syndicale, alors que seulement $2.2 \%$ des conventions visant $1.2 \%$ des travailleurs ne contenaient aucune disposition à ce sujet ${ }^{16}$.

Ainsi qu'on peut le constater, la formule Rand n'est pas aussi généralisée qu'on le laisse croire. La même étude révèle qu'en ce qui regarde l'adhésion obligatoire au syndicat, $32.7 \%$ des conventions visant $35.2 \%$ des travailleurs contenaient une disposition d'atelier syndical parfait ${ }^{17}$ et $20.7 \%$ des conventions couvrant $20.6 \%$ des travailleurs possédaient une clause d'atelier syndical imparfait ${ }^{18}$.

15 Agreement between Ford Motor Company of Canada Limited and International UAW Local 200, 584, 707, 1054 et 1520, Décembre 9, 1973, art. 5 et 8.

16 Disposition de grandes conventions collectives concernant les employés dans l'industrie manufacturière au Canada (1974), Travail Canada, Ottawa, page 5.

17 Voir note 2 pour la définition de l'atelier syndical parfait et la note 14 pour celle de l'atelier syndical imparfait.

18 Ibid., page 4. 